\title{
Resistance of Commercial Bed Covers against Faecal Pellets of House Dust Mites: Behaviour of Seams and Zippers
}

\author{
Dirk Höfer*, Nadja Berner-Dannenmann, Christoph Marquardt, Timo Hammer \\ Hohenstein Institute for Textile Innovation gGmbH, Schloss Hohenstein, Boennigheim, Germany \\ Email: *d.hoefer@hohenstein.de
}

How to cite this paper: Höfer, D., Berner-Dannenmann, N., Marquardt, C. and Hammer, T. (2019) Resistance of Commercial Bed Covers against Faecal Pellets of House Dust Mites: Behaviour of Seams and Zippers. Journal of Textile Science and Technology, 5, 27-37.

https://doi.org/10.4236/jtst.2019.52003

Received: March 13, 2019

Accepted: May 5, 2019

Published: May 8, 2019

Copyright () 2019 by author(s) and Scientific Research Publishing Inc. This work is licensed under the Creative Commons Attribution International License (CC BY 4.0).

http://creativecommons.org/licenses/by/4.0/

(c) (i) Open Access

\begin{abstract}
This study evaluated the allergen impermeability against airborne allergens of dust mite droppings through all parts of commercial bed covers, including surface seams and zippers. Specimens were taken from places with and without seams and zipper. A novel penetration cell was developed to expose the specimens to an inoculum of purified mite droppings that was assessed for its allergen content Der p1 prior to the penetration tests. Using covers of different construction and material, the penetration level increased significantly in the presence of seams and zippers and could reach up to 6\% depending on the seam's/zipper's characteristics and quality. Therefore, zippers and seams have to be considered as access points for the penetration of mite droppings. As for the penetration of airborne mite particles through the zipper, the penetration level was greatly attenuated by the presence of a cover strip. Depending on the respective quality and the construction type, the mite allergen Der 1 penetrated most likely through the zipper and seams of the specimens, already after a single laundry cycle. Hence, laundry may compromise the barrier performance and proves to be an important quality feature. In all samples, the textile surface showed sufficient allergen impermeability. Our conclusions provide recommendations to both manufacturers and users.
\end{abstract}

\section{Keywords}

Dust Mite, Allergen, Dermatophagoides pteronyssinus, Allergy, Laundry, Nonwoven, Fabric, Encasing

\section{Background}

House dust mites (HDM, Dermatophagoides pteronyssinus, of the Pyroglyphidae family) are amongst the most important sources of indoor allergens world- 
wide [1]. Hypersensitivity to house dust mite allergens, a protein of HDM bodies, is one of the most common allergic responses, affecting up to $85 \%$ of asthmatics (primarily Der f1 in the US and Der p1 in Europe and elsewhere). When the mite expels waste, the protein adheres to the mites' feaces and is expelled along with the faecal pellet and finally inhaled, thus triggering the allergic response of HDM patients [2].

Besides residence in allergen-free environments, there are other more suitable methods of allergen avoidance for sufferers at home, many of which are textile-based. As HDM are found in almost all dwellings and in much larger concentrations in mattresses than on floors [3], avoidance of HDM in their beds is crucial for allergic patients since mattresses are the most important habitat and source of mite allergens to which we are exposed for many hours during nocturnal sleep [4]. Especially during movement in bed as well as bedmaking, HDM faeces become airborne and then can easily be inhaled [5]. Each movement of the sleeper results in a cloud of allergen-causing particles that evades the mattress. That is why in 2007 the Expert Panel Report 3, "Guidelines for the Diagnosis and Management of Asthma", recommends to encase mattresses and pillows in allergen-impermeable covers [6]. However, it has not yet been shown that encasement of bedding is associated with clinical benefits [7]. For example, three studies reported that mattress covers without other measures were not effective [8] [9] [10]. Likewise, two well-conducted studies failed to show an effect of HEPA filters alone [11] [12]. The absence of clinical benefits could either be explained by unknown access points of bed covers like poor barrier efficacy of the fabric, the seams or zippers, or for example further allergen sources [7].

Today, there are no guidelines as to the desirable properties for bed covers. When looking at several materials commercially available, the question of quality regarding the HDM protection is often overlooked. Thus, bed covers of various materials and with different construction techniques can be found, most of which focus on the resistance of cover fabric versus HDM and their allergens [13]. The leak rate of covers goes back to work of Vaughan et al., who studied the permeability of woven and nonwoven fabrics by measuring the pressure gradient across the fabric [14]. They recommended that covers with a pore size of 2 - $10 \mu \mathrm{m}$ are suitable for allergy sufferers, because of their ability to block the passage of all HDM droppings, which are assumed to have particle sizes between 10 - $40 \mu \mathrm{m}$ [4]. Hence, most modern commercial bed covers fabrics show pore sizes of $2-10 \mu \mathrm{m}$. Both tight-woven and nonwoven encasement materials have been widely used by patients with some clinical benefit. Some of the covers are film-coated, e.g. by either polyurethane-membranes or plastic, which also provide good protection [15], but are at least comfortable because of air flow limitations and noise emission.

There are comprehensive methodological studies addressing the barrier performance of encasings [13]. However, test approaches in these studies evaluated the resistance of the fabric using either living HDM or dust collections and were run exclusively on samples of the textile surface, to determine the penetration 
level of the cover. To our knowledge, the potential for a permeation of HDM allergens through seam and zipper has not yet been determined, even though these areas would be privileged penetration points for mite droppings. Therefore, this work was designed to evaluate the penetration level, or likewise the allergen impermeability, through all parts of bed covers including seams and zippers. For that purpose, we first established a cultivation of real HDM to develop an inoculum of purified droppings, which was assessed for its Der p1 allergen content. Compared to other test approaches, we also used a novel penetration cell that permits utilisation of seams and zippers. In addition, we wanted to know whether laundry compromises the barrier performance of the bed cover materials.

\section{Material and Methods}

\subsection{Bed Cover Samples}

Three types of commercially available bed cover claiming to provide protection from HDM and their allergens were collected. Tightly woven and nonwovens covers were chosen, as these types dominate the markets worldwide. All covers were obtained directly from the manufacturer. The materials and constructions of the samples were analysed by stereomicroscopy (Olympus SZX12, Hamburg, Germany) and then classified into: Brand A, a PU-membrane coated tightly woven fabric 50\% PES/50\% CO cotton with double-stitched seams and lapped nylon coil zippers; brand B, a non-coated tight nonwoven made of 70\% PES, 30\% PA microfibres with reinforced seams and a two-sided plastic zipper with a cover strip; and brand $\mathrm{C}$ consisting of a non-coated tight nonwoven, made of $70 \%$ PES, 30\% PA microfibres with seams presenting stab stitches and a moulded plastic zipper. Samples were cut into test specimens of $15 \times 15 \mathrm{~cm}^{2}$ rectangles. To confirm the validity of the test system, an in-house reference fabric made of 100\% PA 6.6 (SEFAR Nitex 03-15/10, SEFAR AG, Thal, Switzerland) with a defined $15 \mu \mathrm{m}$ mesh opening and $10 \%$ open area was included as negative control specimen in all tests. A plastic cover served as a positive control.

\subsection{HDM Stock Cultures and Cultivation of HDM Faeces}

HDM, D. pteronyssinus, were cultivated in-house on a substrate mixture of nutrient, vitamins and minerals (particle size $<1 \mathrm{~mm}$ ). They were constantly kept in a desiccator at a temperature of $25^{\circ} \mathrm{C} \pm 1{ }^{\circ} \mathrm{C}$ and a relative humidity of $75 \% \pm$ $1 \%$ in the dark in small glass vials with a semi-permeable lid. For continuous cultivation, mites were subcultured after $6-8$ weeks by adding one microspoon of the old HDM culture to $100 \mathrm{mg}$ of fresh HDM feed.

After 8 weeks of cultivation, feed and HDM culture had turned into aged mite droppings, a composite faeces sample referred to as HDM faeces. Dead bodies of HDM were combed out by sieving through a $200 \mu \mathrm{m}$ mesh and HDM faeces stored at $-28^{\circ} \mathrm{C}$ until use. Prior to use, HDM faeces was acclimatised at room temperature. For scanning electron microscopy, HDM faeces was dried, mounted on metal stubs, covered with an Ion Sputter SCD/040 instrument for examina- 
tion and analysed with a scanning electron microscope (Jeol JSM 6100, Jeol Ltd., Tokio, Japan) set at 5 and $10 \mathrm{kV}$.

Prior to the penetration tests, the allergen amount of HDM faeces was assessed via a ELISA-immunoassay to evaluate its total content of Der p1 allergen (Indoor Biotechnologies, Warmister, UK). This was done using a spectrophotometer (TecanGenios Reader, Crailsheim, Germany) set at $405 \mathrm{~nm}$.

\subsection{Penetration Tests of Bed Covers}

$15 \times 15 \mathrm{~cm}^{2}$ rectangles of the test specimens were clamped in a metal sample holder (penetration cell) with a clamping ring. Then HDM faeces were placed on the top of the test specimens. All specimens were mounted with their inner surface in a test chamber (penetration cell) that was exposed to HDM faeces to test for the specimens' resistance for mite droppings. Following this, specimens were incubated for 8 hours (to simulate nocturnal sleep) under a pressure of $20.6 \mathrm{~Pa}$ with continuous agitation using ceramic marbles. Afterwards, all particles of mite droppings that permeated through the specimens were carefully eluted in 3 $\mathrm{ml}$ PBS-Tween to check for their Der p1 level by immunoassay (Indoor Biotechnologies, Warmister, UK). Extracts were stored at $4^{\circ} \mathrm{C}+/-2^{\circ} \mathrm{C}$ overnight until measurement. All specimens, fabrics, seams and zippers, were tested in triplicates. Statistical analysis was performed using MS Excel (Microsoft Corporation, USA). Mean values of these measurements were taken to calculate the penetration level according to the formula:

Total content of Der p1 recovered below specimen divided by total content of Der $\mathrm{p} 1$ of HDM faeces load, multiplied by $100=$ penetration level in $\%$. The percentage of allergen impermeability [\%] (i.e. barrier effect to allergen) was calculated by subtraction: $100 \%$ minus penetration level.

\subsection{Laundry}

Bed covers were also subjected to a domestic laundry cycle at $60^{\circ} \mathrm{C}$. Laundry was carried out in a Linitest according to ISO-105-C06-A1S at $60^{\circ} \mathrm{C}$ for $30 \mathrm{~min}$. As detergent solution, the standard IEC detergent was used $(4.0 \mathrm{~g} / \mathrm{l} ; 150 \mathrm{ml}$ per sample). Disinfectants, chlorine-based products as well as fabric softeners and ballast load were omitted. After laundry, all bed covers were rinsed twofold with $\mathrm{H}_{2} \mathrm{O}$ for 2 min and finally air-dried.

\section{Results}

The homogeneity and composition of HDM faeces was tested by scanning electron microscopy. Fresh mite droppings showed typical diameters of approx. 2 $\mu \mathrm{m}$, however after several weeks of cultivation, dropping diameters decreased. Thus, ageing of the HDM culture and mite droppings over 8 weeks resulted in a wide range of typical round mite droppings, showing diameters of $0.3 \mu \mathrm{m}$ up to $>2 \mu \mathrm{m}$. A typical characteristic after 8 weeks of cultivation is depicted in Figure 1. Droppings could easily be distinguished from the few and more irregularly 


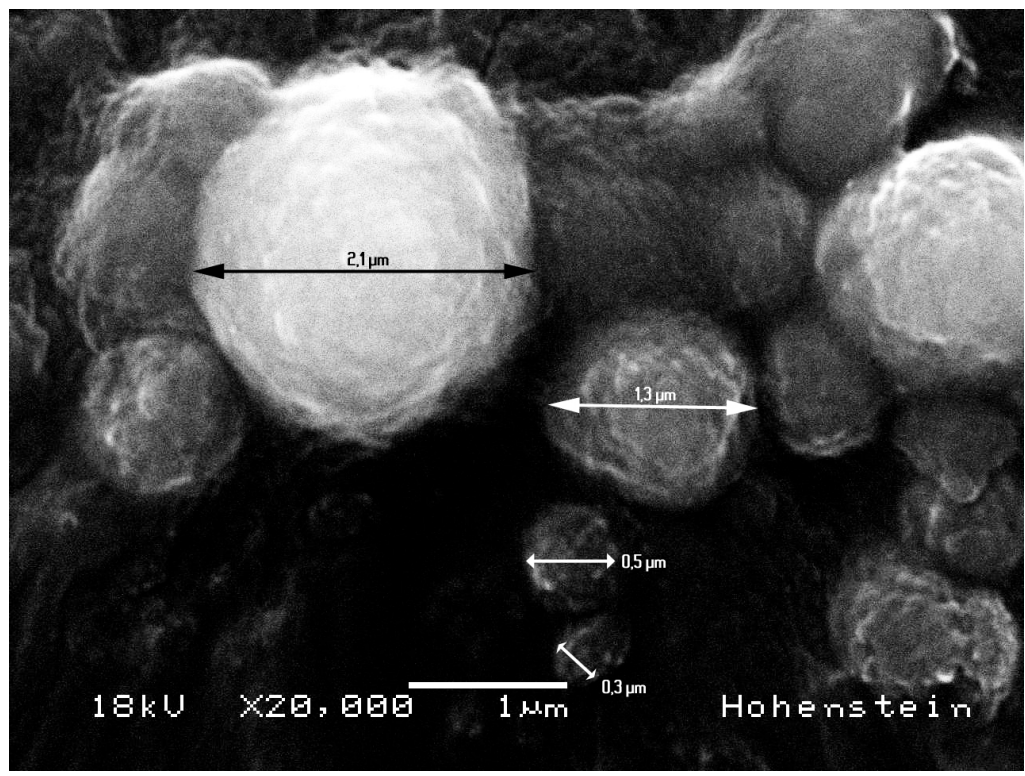

Figure 1. Scanning electron microscopy of standardised HDM faeces. Please note range in diameters of dust mite droppings, some of which are below $2.1 \mu \mathrm{m}$.

shaped remnants of HDM feed, which was more or less depleted. Using a stereomicroscope, we found all HDM dead (not shown). HDM faeces was also analysed for its Der p1 content by immunoassay. A typical allergen amount applied per test specimen was in the range of $10-12 \mu \mathrm{g}$ Der p1 present in $8-12 \mathrm{mg}$ of HDM faeces, depending on the respective HDM cultivation.

After the morphological and immunological characterisation for its allergen content, HDM faeces containing a pre-determined content of Der p1 was used to test the barrier effect of bed covers using the novel penetration cell. Table 1 presents the degree of allergen impermeabilities with regard of mean percentages of the test specimens. The internal control fabric showed a constant reproducible allergen impermeability due to its defined structure with an open area of $10 \%$ and $15 \mu \mathrm{m}$ mesh openings, whereas the plastic cover blocked all airborne particles (see Table 1). In the new state of the bed cover samples, we observed a high barrier effect of around $99 \%$ with all specimens of fabric surfaces, i.e. the membrane-coated tight woven and the two nonwovens. When the bed covers were washed just once, the allergen impermeabilities of the textile surfaces of brand A and B did not attenuate, with the exemption of brand C, that slightly dropped in its barrier for mite droppings to $95.64 \%$. In its new state, brand C also offered only minimal protection for the allergen load at its seams (penetration level 3.48\%) and zipper (penetration level 4.1\%). In the new state, the seam and zipper constructions of brand A and B showed optimal protection by reaching approximately the starting values for allergen impermeability of their respective fabric surfaces ( $99 \%)$. In another set of experiments, the cover strip for the zipper of brand B was put down to cover the zipper according to the recommendations of the manufacturer (see Figure 2(C)). However, when the cover strip of the zipper of brand B was flipped over to expose the zipper directly 
Table 1. Mean allergen impermeabilities and penetration levels of bed covers test specimens. Test specimens are surfaces, seams and zippers.

\begin{tabular}{cccccc}
\hline Type of fabric & Allergen & Penetration & Type of fabric & Allergen & Penetration \\
\hline new state & impermeability \% & level \% & washed & impermeability \% & level \% \\
\hline A/surface & 99.84 & 0.16 & A/surface & 99.21 & 0.79 \\
A/seam & 99.75 & 0.25 & A/seam & 98.62 & 1.38 \\
A/zipper & 99.71 & 0.29 & A/zipper & 97.67 & 2.33 \\
B/surface & 99.90 & 0.10 & B/surface & 99.57 & 0.43 \\
B/seam & 99.88 & 0.12 & B/seam & 99.49 & 0.51 \\
B/zipper & 99.75 & 0.25 & B/zipper & 99.29 & 0.71 \\
B/flipped zipper & 98.22 & 1.78 & B/flipped zipper & 97.51 & 2.49 \\
C/surface & 98.98 & 1.02 & C/surface & 95.64 & 4.36 \\
C/seam & 96.52 & 3.48 & C/seam & 93.99 & 6.01 \\
C/zipper & 95.90 & 4.10 & C/zipper & 93.16 & 6.84 \\
plastic cover & 100 & 0 & plastic cover & 100 & 0 \\
\hline
\end{tabular}
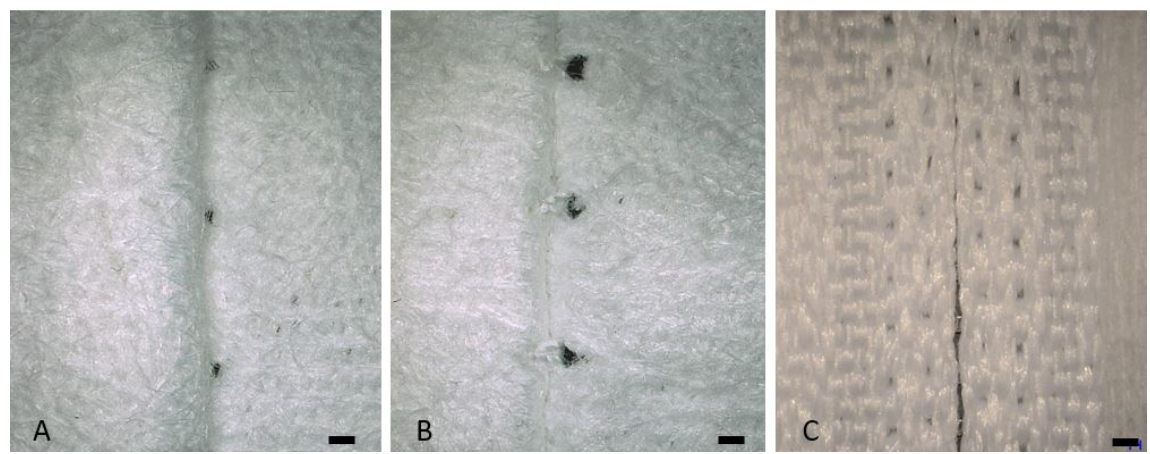

Figure 2. Stereomicroscopy of brand C seams in new state (A) and washed (B). Section of C shows covered zipper of brand B. Bars $0.6 \mathrm{~mm}$.

to HDM faeces load, we found a penetration level of $1.78 \%$ in the new state. Thus, for the penetration of airborne mite particles through the zipper, the penetration level was greatly attenuated by the presence of a cover strip. However, the zippers penetration level of brand B was further increased by a single washing cycle. In these experiments, the flipped zipper achieved a value of $2.49 \%$.

Domestic washing also reduced the barrier efficacy of seams and zippers of brand $\mathrm{A}$ and $\mathrm{C}$. The barrier against HDM faeces of brand $\mathrm{C}$ seams and zippers reached a penetration level of more than $6 \%$ from the starting value of the positive control. Stereomicroscopy revealed that a single laundry cycle expanded the stitching holes of the seam of brand $C$ when it was lightly stretched (see Figure 2(A), Figure 2(B)). In contrast to this, the seam of brand A at least achieved an allergen impermeability of $98.62 \%$ whereas the specimen exposing the zipper displayed a barrier effect of $97.67 \%$.

\section{Discussion}

This study was run to explore the resistance of the textile surfaces, seams and zippers of allergen-impermeable bed covers with regard to their ability to block 
purified HDM droppings. To our knowledge, at this point no test approach has been set up which separately assesses each single component of bed covers for its barrier effect to block the passage of allergens. In contrast to other test methods which strain samples using house dust endowed with HDM spent medium [13], we thought to stress textile surfaces, seams and zippers with an inoculum of purified mite droppings with a standardised Der p1 content. Morphological characterisation revealed that HDM faeces aged over 8 weeks displayed droppings of diverse sizes, showing diameters between $0.3 \mu \mathrm{m}$ up to $>2 \mu \mathrm{m}$. However, smaller droppings constituted a much smaller fraction than the larger particles (approx. 2\%, not shown). This finding is in contrast to previous studies, which categorised mite feces as fine particles in domestic dust ranging in size from 10 $40 \mu \mathrm{m}[4]$ [14], which is the typical size of fresh faeces [4] [14]. The difference in size may be explained by disintegration of the droppings due to ageing of the HDM faeces. Given the fact that ageing, disintegration of droppings and reduction in size may be a process that naturally also occurs in infested mattresses, this could have consequences as to reconsider the recommendations for bed cover constructions.

Comprehensive work has been done to assess the efficacy of encasing materials against HDM and their allergens [15]. Vaughan et al. evaluated materials used for bedding encasements and found that tightly woven fabrics and nonwovens fabrics can block HDM allergens but still allow some airflow for comfort aspects [14]. These covers were also found to show best prevention of living mite penetration using a special Siriraj chamber [15]. As this chamber cannot measure the allergens in mite faeces [16], we thought to construct a novel test chamber that allows to investigate all components of bed covers and also allows to extract the level of allergen that penetrated the specimens.

Regardless of the inoculum with average smaller dropping pore sizes, we found that in their new state the textile surfaces of all bed covers afforded excellent allergen impermeabilities of approximately $99 \%$. Since all samples showed pore sizes between $2-6 \mu \mathrm{m}$, as recommended by Vaughan et al. [14], they were able to prevent the passage of the purified HDM load. However, nonwoven brand C slightly dropped in its barrier for mite allergens to $95.64 \%$ when the textile surface underwent a single laundry cycle. Given the fact, that both nonwoven samples were made from identical fibre blends, and that brand B was able to maintain its allergen impermeability after washing, we assume that quality aspects like different fibre bonding of brand $\mathrm{C}$ account for these barrier differences [17]. Our findings underline the recommendations of some manufacturers of nonwoven encasings, which prohibit washing of their covers.

Laundry also increased the penetration level of allergens when seams and zippers of brand $\mathrm{C}$ were exposed to purified HDM faeces. Compared to the quality of the seams and zipper of brand $\mathrm{B}$, brand $\mathrm{C}$ achieved an increase of $6 \%$ in its penetration level against a comparable allergen load, whereas seams and zipper of the woven fabric brand A afforded better after washing the sample. At this point it should be noted that a penetration level of $6 \%$ in our test setup, ex- 
pressed in absolute values, is equal to a penetration of approximately $600 \mathrm{ng}$ Der $\mathrm{p} 1$ over a time period of $8 \mathrm{~h}$ and in relation to a total allergen load of $10 \mu \mathrm{g}$. It should also be kept in mind that this amount is more than a quarter of the threshold level discussed by experts (e.g. First International Workshop on Mite Allergens and Asthma) [6]. Current studies seeking to quantify a level of exposure that can be considered "safe" suggest that HDM allergens levels of less than 2 $\mu \mathrm{g} / \mathrm{g}$ Der $\mathrm{p} 1$ are the maximum level for the primary prevention of sensitization which should also avoid an acute attack of asthma [18]. Based on these considerations and our present findings, zippers and seams have to be considered as entry points for the penetration of mite allergens, especially as their respective penetration levels have to sum up. In our hands, the washed brand $\mathrm{C}$ specimens accounted for a total penetration level of $17.21 \%$, which would expose a hypersensitive patient to more than $2 \mu \mathrm{g}$ of Der p1 during a single $8 \mathrm{~h}$ rest on a covered mattress. Therefore, construction and quality of these materials together with wash resistance should be defined in future guidelines for improved barrier function of bed covers.

A study run by Mahakittikun et al. showed that nonwoven covers exhibited worn and matted surface threads that easily provided access points for mite penetration [16]. Hence it is reasonable to assume that those structural changes might also affect the allergen resistance of other cover constructions, especially after repetitive laundering or use over time. To study the effect of washing on the bed covers allergen resistance, we ran a single domestic laundry cycle followed by a second set of resistance tests. A single domestic washing cycle diminished the allergen impermeability of two of the samples. In particular, we concluded from our experiments, that washing reduced the structural integrity of seams and zippers, e.g. by deterioration and loosening of fibers of the seams and the inserted zipper, thereby creating access points for penetration of mite droppings. It is widely known that the seams performance and quality depend on various factors such as seam strength, seam slippage, seam puckering, seam appearance and the yarn [19]. Up to now, the effect of laundry on bed covers has only been done with respect to the removal of allergens. For example, Arlian et al. studied the mite allergen removal during machine washing of laundry and found cold or warm water was effective and removed most allergen [20]. However, despite the fact that mite allergens are quite soluble in water and washing methods have been shown to remove most of dust and allergen [21] [22], modern manufacturers seldom recommend washing bed covers, presumably as they are aware that mechanical and chemical stress strains the functionality of the fabric.

Recently, Tovey et al. run a time-based measurement of personal mite allergen bioaerosol exposure over 24 hour periods using a novel sampling to assess daily exposure of HDM allergic patients [23]. They found that exposure pattern varied over time with indoor domestic exposure accounting for nearly $60 \%$ of total exposure. Total in-bed-asleep exposure accounted for about $10 \%$ of total daily exposure, suggesting that beds are not the main site of exposure. Their finding is 
somewhat inconsistent to observations by patients who especially face hypersensitivity reactions during sleep. Although various studies showed that mattress bed covers reduce HDM allergen concentrations [24], several Cochrane studies observed that HDM avoidance did not show a protective effect on the development of sensitisation to HDM or symptomatic allergy in children up to age 24 month [25] or adults [7] [26]. The non-significant effects of bed covers on clinical parameters of bed covers could be explained by three of our findings: First, if droppings of HDM naturally age and mature in mattresses, followed by disintegration into much smaller parts, they might become airborne and available to patients via seams and zippers of lower quality. Future studies will have to strengthen this point. Second, laundry of bed covers might further attenuate the barrier efficacy of seams and zippers. Lastly, we found that flipping a cover strip to mask the zipper significantly improved the zippers allergen impermeability. Thus, bed covers should all be equipped with a covered zipper and patients should take care not to unflip it in daily use.

It may be concluded from our findings, that the textile surface of membrane-coated wovens and tightly packed nonwovens prevent the leakage of mite droppings, while the seams and zippers of covers are sufficiently lacking in this regard, depending on their respective construction and quality. For the choice of an effective encasing product user should be aware, that seams and zippers are access points. Manufacturer thus should reinforce seams and zippers especially with regard to washing the bed covers without reducing its barrier effect. It is also advisable to construct covers with pore sizes smaller than $2 \mu \mathrm{m}$ as mite droppings may disintegrate over time into much smaller particles. Other quality aspects of bed covers to be considered for a future guideline are: Air permeability, water vapour resistance, noise emission and wash resistance.

\section{Conclusion}

In this study, we found significant differences in the barrier efficacy of three common encasing types of different price and quality ranges, mainly in the zipper and seam area. Our results show that zippers and seams have to be considered as access points for the penetration of mite droppings, especially as HDM faeces disintegrate over time into small particles with diameters between $0.3 \mu \mathrm{m}$ up to $>2 \mu \mathrm{m}$. Laundry may compromise the barrier performance and thus proves to be an important quality feature. These conclusions provide recommendations to both manufacturers and users. Future guidelines for consumers on dust-mite covers should include specifications towards laundry, design and construction that are based on quantitative data for allergen penetration levels of fabric, seam and zipper rather than to prove anti-mite or mite-penetration properties.

\section{Acknowledgements}

The authors gratefully thank all technical assistants from Hohenstein involved in this study. The study received no financial support. The authors have no finan- 
cial or other conflict of interest to report.

\section{Funding}

The research was not written as a project, so no research funding was requested.

There is no funding statement of this paper.

\section{Conflicts of Interest}

There is no conflict of interest regarding the publication of this paper.

\section{References}

[1] Pawankar, R., Canonica, G., Holgate, S., et al. (2011) World Allergy Organization (WAO) White Book on Allergy. World Allergy Organisation, Wisconsin. https://doi.org/10.1097/WOX.0b013e318238f58f

[2] Custovic, A. and Simpson, A. (2012) The Role of Inhalant Allergens in Allergic Airways Disease. Journal of Investigational Allergology and Clinical Immunology, 22, 393.

[3] Maunsell, K., Wraith, D. and Cunnington, A.M. (1968) Mites and House-Dust Allergy in Bronchial Asthma. The Lancet, 291, 1267-1270.

https://doi.org/10.1016/S0140-6736(68)92289-7

[4] Tovey, E.R., Chapman, M. and Platts-Mills, T. (1981) Mite Faeces Are a Major Source of House Dust Allergens. Nature, 289, 592. https://doi.org/10.1038/289592a0

[5] Platts-Mills, T.E., Mitchell, E.B., Nock, P., et al. (1982) Reduction of Bronchial Hyperreactivity during Prolonged Allergen Avoidance. The Lancet, 320, 675-678. https://doi.org/10.1016/S0140-6736(82)90709-7

[6] Busse, W., Boushey, H., Camargo, C., et al. (2007) Expert Panel Report 3: Guidelines for the Diagnosis and Management of Asthma. US Department of Health and Human Services, National Heart Lung and Blood Institute, Washington DC, 1-417.

[7] Gøtzsche, P.C. and Johansen, H.K. (2008) House Dust Mite Control Measures for Asthma: Systematic Review. Allergy, 63, 646-659. https://doi.org/10.1111/j.1398-9995.2008.01690.x

[8] Luczynska, C., Tredwell, E., Smeeton, N., et al. (2003) A Randomized Controlled Trial of Mite Allergen-Impermeable Bed Covers in Adult Mite-Sensitized Asthmatics. Clinical \& Experimental Allergy, 33, 1648-1653. https://doi.org/10.1111/j.1365-2222.2003.01729.x

[9] Terreehorst, I., Hak, E., Oosting, A.J., et al. (2003) Evaluation of Impermeable Covers for Bedding in Patients with Allergic Rhinitis. New England Journal of Medicine, 349, 237-246. https://doi.org/10.1056/NEJMoa023171

[10] Woodcock, A., Forster, L., Matthews, E., et al. (2003) Control of Exposure to Mite Allergen and Allergen-Impermeable Bed Covers for Adults with Asthma. New England Journal of Medicine, 349, 225-236. https://doi.org/10.1056/NEJMoa023175

[11] Wood, R.A., Johnson, E.F., Van Natta, M.L., et al. (1998) A Placebo-Controlled Trial of a HEPA Air Cleaner in the Treatment of Cat Allergy. American Journal of Respiratory and Critical Care Medicine, 158, 115-120. https://doi.org/10.1164/ajrccm.158.1.9712110

[12] Francis, H., Fletcher, G., Anthony, C., et al. (2003) Clinical Effects of Air Filters in Homes of Asthmatic Adults Sensitized and Exposed to Pet Allergens. Clinical \& Experimental Allergy, 33, 101-105. 
https://doi.org/10.1046/j.1365-2222.2003.01570.x

[13] Mahakittikun, V., Boitano, J.J., Komoltri, C., et al. (2009) Anti-Mite Covers: Potential Criteria for Materials Used against Dust Mites. Textile Research Journal, 79, 436-443. https://doi.org/10.1177/0040517508093417

[14] Vaughan, J.W., McLaughlin, T.E., Perzanowski, M.S., et al. (1999) Evaluation of Materials Used for Bedding Encasement: Effect of Pore Size in Blocking Cat and Dust Mite Allergen. Journal of Allergy and Clinical Immunology, 103, 227-231. https://doi.org/10.1016/S0091-6749(99)70495-1

[15] Mahakittikun, V., Komoltri, C., Nochot, H., et al. (2003) Laboratory Assessment of the Efficiency of Encasing Materials against House Dust Mites and Their Allergens. Allergy, 58, 981-985. https://doi.org/10.1034/j.1398-9995.2003.00095.x

[16] Mahakittikun, V., Boitano, J.J., Tovey, E., et al. (2006) Mite Penetration of Different Types of Material Claimed as Mite Proof by the Siriraj Chamber Method. Journal of Allergy and Clinical Immunology, 118, 1164-1168. https://doi.org/10.1016/j.jaci.2006.07.025

[17] Mahakittikun, V., Jirapongsananuruk, O., Nochot, H., et al. (2003) Woven Material for Bed Encasement Prevents Mite Penetration. Journal of Allergy and Clinical Immunology, 112, 1239-1241. https://doi.org/10.1016/j.jaci.2003.08.045

[18] Calderón, M.A., Linneberg, A., Kleine-Tebbe, J., et al. (2015) Respiratory Allergy Caused by House Dust Mites: What Do We Really Know? Journal of Allergy and Clinical Immunology, 136, 38-48. https://doi.org/10.1016/j.jaci.2014.10.012

[19] Bharani, M., Shiyamaladevi, P. and Mahendra Gowda, R. (2012) Characterization of Seam Strength and Seam Slippage on Cotton Fabric with Woven Structures and Finish. Research Journal of Engineering Sciences, 1, 41-50.

[20] Arlian, L.G., Vyszenski-Moher, D.L. and Morgan, M.S. (2003) Mite and Mite Allergen Removal during Machine Washing of Laundry. Journal of Allergy and Clinical Immunology, 111, 1269-1273. https://doi.org/10.1067/mai.2003.1547

[21] McDonald, L.G. and Tovey, E. (1992) The Role of Water Temperature and Laundry Procedures in Reducing House Dust Mite Populations and Allergen Content of Bedding. Journal of Allergy and Clinical Immunology, 90, 599-608. https://doi.org/10.1016/0091-6749(92)90132-L

[22] Siebers, R.W., Patchett, K., Fitzharris, P., et al. (1996) Mite Allergen (Der p 1) on Children's Clothing. Journal of Allergy and Clinical Immunology, 98, 853-854. https://doi.org/10.1016/S0091-6749(96)70141-0

[23] Tovey, E.R., Liu-Brennan, D., Garden, F.L., et al. (2016) Time-Based Measurement of Personal Mite Allergen Bioaerosol Exposure over 24 Hour Periods. PLoS ONE, 11, e0153414. https://doi.org/10.1371/journal.pone.0153414

[24] Halken, S., Høst, A., Niklassen, U., et al. (2003) Effect of Mattress and Pillow Encasings on Children with Asthma and House Dust Mite Allergy. Journal of Allergy and Clinical Immunology, 111, 169-176. https://doi.org/10.1067/mai.2003.5

[25] Horak, F., Matthews, S., Ihorst, G., et al. (2004) Effect of Mite-Impermeable Mattress Encasings and an Educational Package on the Development of Allergies in a Multinational Randomized, Controlled Birth Cohort Study-24 Months Results of the Study of Prevention of Allergy in Children in Europe. Clinical \& Experimental Allergy, 34, 1220-1225. https://doi.org/10.1111/j.1365-2222.2004.02024.x

[26] Nankervis, H., Pynn, E.V., Boyle, R.J., et al. (2015) House Dust Mite Reduction and Avoidance Measures for Treating Eczema. Cochrane Database of Systematic Reviews, 1, CD008426. https://doi.org/10.1002/14651858.CD008426.pub2 